\title{
Evidence and knowledge gaps on the disease burden in sexual and gender minorities: a review of systematic reviews
}

\author{
Karel Blondeel ${ }^{1 *}$, Lale Say ${ }^{2}$, Doris Chou², Igor Toskin²,3 Rajat Khosla², Elisa Scolaro ${ }^{2}$ and Marleen Temmerman ${ }^{1,2}$
}

\begin{abstract}
Sexual and gender minorities (SGM) include individuals with a wide range of sexual orientations, physical characteristics, and gender identities and expressions. Data suggest that people in this group face a significant and poorly understood set of additional health risks and bear a higher burden of some diseases compared to the general population. A large amount of data is available on HIV/AIDS, but far less on other health problems. In this review we aimed to synthesize the knowledge on the burden of communicable and non-communicable diseases, mental health conditions and violence experienced by SGM, based on available systematic reviews. We conducted a global review of systematic reviews, including searching the Cochrane and the Campbell Collaboration libraries, as well as PubMed, using a range of search terms describing the populations of interest, without time or language restrictions. Google Scholar was also scanned for unpublished literature, and references of all selected reviews were checked to identify further relevant articles. We found 30 systematic reviews, all originally written in English. Nine reviews provided data on HIV, 12 on other sexually transmitted infections (STIs), 4 on cancer, 4 on violence and 3 on mental health and substance use. A quantitative meta-analysis was not possible. The findings are presented in a narrative format. Our review primarily showed that there is a high burden of disease for certain subpopulations of SGM in HIV, STIS, STI-related cancers and mental health conditions, and that they also face high rates of violence. Secondly, our review revealed many knowledge gaps. Those gaps partly stem from a lack of original research, but there is an equally urgent need to conduct systematic and literature reviews to assess what we already know on the disease burden in SGM. Additional reviews are needed on the non-biological factors that could contribute to the higher disease burden. In addition, to provide universal access to health-care for all, more information is needed on the barriers that SGM face in accessing health services, including the attitudes of health-care providers. Understanding these barriers and the additional health risks they impose is crucial to improving the health status of SGM.
\end{abstract}

Keywords: Sexual and gender minorities, Disease burden, LGBT, Health

\section{Review}

\section{Background}

The Constitution of the World Health Organization (WHO) states that the "enjoyment of the highest attainable standard of health is one of the fundamental rights of every human being without distinction of race, religion, political belief, economic or social condition" [1]. Although medical ethics require health services to be inclusive of all people, some populations have more

\footnotetext{
* Correspondence: karel.blondeel@ugent.be

${ }^{1}$ Ghent University, Ghent, Belgium

Full list of author information is available at the end of the article
}

difficulties than others in reaching this standard of health [2]. Those populations can be defined by age, gender, race or ethnicity, geography, wealth, disability, and also by sexual orientation and gender identity.

Populations defined by sexual orientation and gender identity include individuals with a wide range of sexual orientations, physical characteristics (as in intersex persons), gender identities and expressions, all of which they may experience differently depending on societal gender norms and cultural traditions. For the purposes of this article we have grouped men who have sex with men (MSM), women who have sex with women (WSW), 
transgender and intersex persons, all defined by the Joint United Nations Programme on HIV and AIDS (UNAIDS), together under the name of 'sexual and gender minorities' or SGM [3]. For research, health policy and advocacy reasons, estimating the size of populations of interest is important [4]. Due to stigma associated with homosexuality and non-gender-normative behaviours, estimates of SGM populations are scarce, but studies show that SGM comprise substantial proportions of the population in countries worldwide [5-7].

Data suggest that SGM face a significant and poorly understood set of additional health risks and bear a higher burden of some diseases compared to the general population $[8,9]$. There have been international calls to answer the unique health-care needs of SGM $[10,11]$. It is therefore indispensable to understand where we are with the knowledge on the health burden of SGM. In 2002 Boehmer showed that research was limited in scope, concentrating mostly on HIV and STIs and MSM [12]. In high-income countries the research scope has been broadening since, but the situation in middle- and low-income countries is less clear [13]. In this review we aimed to collate the knowledge and identify the knowledge gaps on the burden of communicable and noncommunicable diseases, mental health conditions and violence experienced by SGM globally, based on available systematic reviews.

\section{Methods}

A review of systematic reviews can aim to bring together a series of reviews on an important topic or summarize reviews on a variety of topics with a common main theme [14]. This review is of the latter type and while it is unlikely to be of interest to clinicians and patients deciding how best to address a specific problem, it may be relevant to policy makers or to addressing questions that cut across the different reviews [15]. The outcome of a review of reviews is typically a summary of review results. Conducting additional meta-analysis in a review of systematic reviews is a major challenge due to the heterogeneity of the methods, analyses and results of the original reviews. Therefore meta-analysis is not a relevant method to this review of systematic reviews and is descriptive in nature.

\section{Search strategy}

We conducted a review of systematic reviews in concordance with the PRISMA statement [16]. The Cochrane Database of Systematic Reviews and the Campbell Collaboration Library of Systematic Reviews were searched using the following terms to identify reviews on the populations of interest: 'gay,' 'lesbian,' 'transgender,' 'WSW', 'women who have sex with women', 'MSM', 'men who have sex with men', 'same sex', 'sexual orientation,' 'gender identity', 'sexual minority,' 'intersex' and 'homosexuality'. We searched PubMed for the terms 'systematic' AND 'review' combined with a search strategy (see Additional file 1) for sexual and gender minorities (SGM). No language, time or geographical restrictions were applied. The concept of 'burden of disease' was not specified in search strategies. Google Scholar was searched in English and references of the included reviews were checked to identify further relevant articles.

\section{Inclusion and exclusion criteria}

Figure 1 shows how publications were selected for review. Our initial search generated 399 publications. Based on a review of the abstracts, the following exclusions were made: duplicates, one publication lacking an abstract, one protocol of a systematic review, publications that were not systematic reviews, publications without data on disease burden, and publications without data on SGM or with data on only a very specific subset of SGM. We then retrieved and read the full text of 50 retained publications. Those were all publications we found on PubMed, the search on the Cochrane Database of Systematic Reviews and the Campbell Collaboration Library of Systematic Reviews did not generate reviews of interest. Next, we further excluded national reviews if they were included in larger reviews, and reviews that were redundant due to newer published reviews on the same subject and population. Twenty-five articles were retained. An additional five eligible reviews were identified from references and Google Scholar, bringing the total to 30 .

\section{Quality assessment}

The quality of the systematic reviews was assessed with the AMSTAR scale (see Additional file 2) (http:// amstar.ca/). AMSTAR stands for A Measurement Tool to Assess Systematic Reviews and was initially developed to assess systematic reviews of randomised controlled trials [17]. However, it is increasingly being used to assess those that include observational studies as well. The scale consists of 11 items. We considered systematic reviews with a score from 0 to 3 as low quality, from 4 to 6 as medium quality, and from 7 up as high quality. The AMSTAR score was not used as an exclusion criterium, but used as an analytic tool. Regardless of the quality, we did not find inconsistencies in the conclusions of the included individual reviews.

\section{Data extraction}

We created a master table in Microsoft Excel containing key information from each of the included reviews (see Additional file 2): health topic, time range of the search, year published, geographical scope, databases searched, the SGM subpopulation, number of studies included, measures, comparison groups, a summary of the 


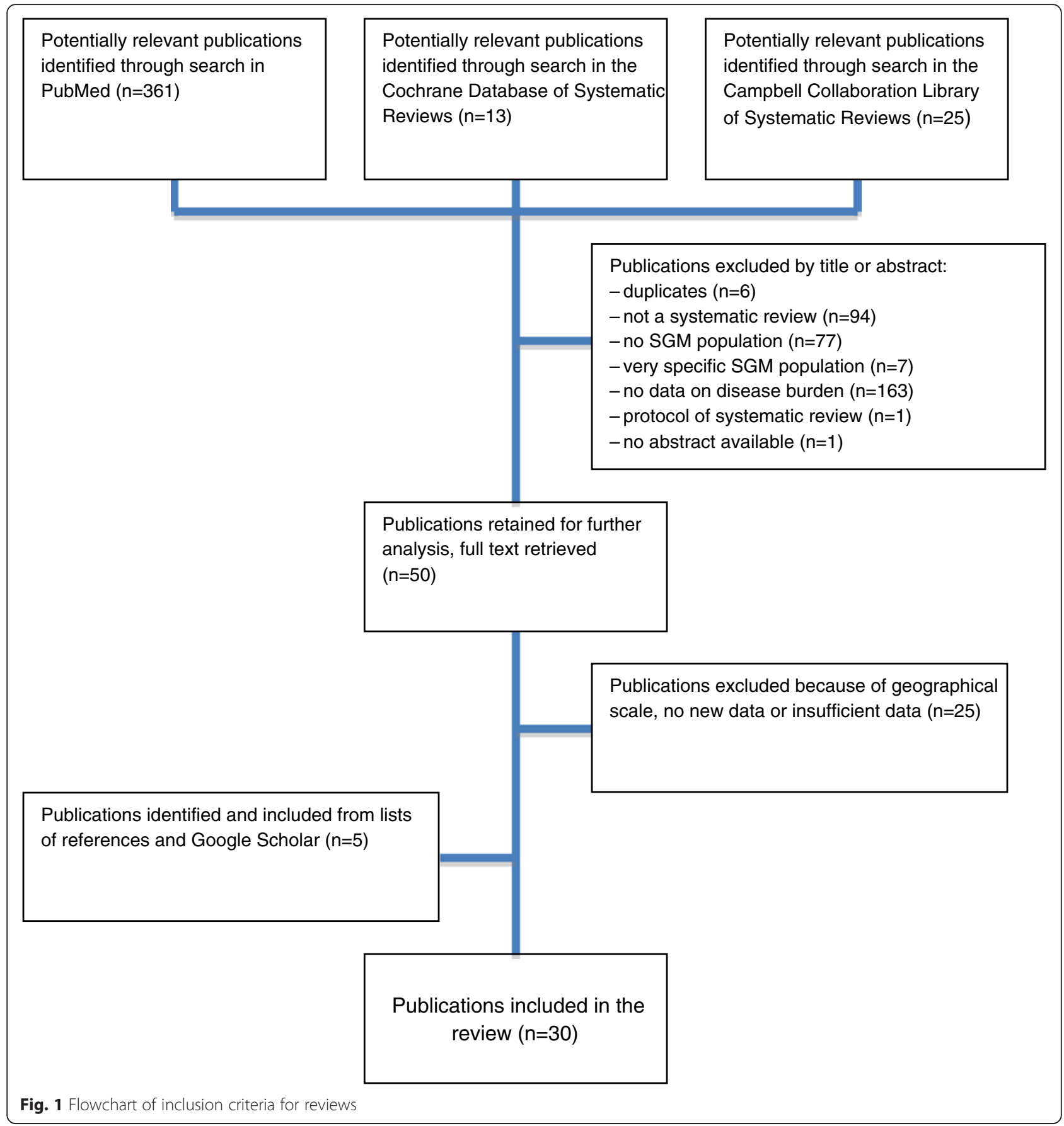

quantitative and/or qualitative data on disease burden and quality assessment score.

\section{Results}

All 30 reviews were originally written in English. Fifteen reviews were global in scope, while 11 were regional and 5 were national. Nineteen reviews were exclusively or predominantly based on data from high-income countries. Nine reviews provided data on $\operatorname{HIV}[6,18-25], 12$ on other sexually transmitted infections (STIs) [21, 26-36], 4 on violence [37-40], 4 on cancer [32, 41-43] and 3 on mental health and substance use [44-46]. One review provided data on both HIV and STIs [21]; another yielded data on STIs as well as cancer [32]. Twenty-five reviews provided data on MSM, 8 on WSW and 5 on transgender persons (some reviews included more than one subpopulation). Twenty-four studies compared data to the general population or other groups, of which one half on the basis of meta-analysis and the other half based on qualitative observations. Fourteen reviews were considered high 
quality $[6,19,20,22,23,26,27,32,36,38,42,44-47], 9$ medium quality $[18,24,30,31,34,35,37,39,40]$ and 7 low quality $[21,25,28,29,33,41,43]$. We found no reviews on intersex people. Detailed results are presented in narrative form under five health topic sub-sections, by SGM subpopulation, as shown in Table 1.

\section{HIV/AIDS}

Nine systematic reviews assessed the burden of HIV in SGM, including 5 global and 4 regional reviews [6, 18-25]. Six of these reviews were on MSM, 2 were on male-tofemale transgender persons and 1 was on WSW.

Men who have sex with men (MSM) Among the 6 reviews that provided data on MSM, 3 were exclusively on MSM. One review was global [24] and 5 were regional $[6,18,19,23,25]$, of which 4 were mainly on low- and middle-income countries (LMICs). Except for one review from 2007, all were published in 2010 or later. All reviews showed a high burden of HIV in MSM. The region with the highest HIV prevalence in MSM was the Caribbean with $25.4 \%$, followed by sub-Saharan Africa with $17.9 \%$ [24]. In contrast, some countries in the Middle East and North Africa show very low HIV prevalence in MSM [6]. In Asia, high rates of incident HIVinfections in MSM were observed wherever studied [18], and across the region of Latin America and the Caribbean MSM was the population most affected by HIV [19]. MSM in LMICs have on average a 19.3 times higher chance of being infected with HIV compared with the general population [23]. In high-income countries, Sullivan et al. showed that among new HIV cases, the proportion attributed to male-male sex increased in all the included countries between 2006 and 2011 [25].

Transgender persons Two systematic reviews, both of high quality, provided data on HIV infection in male-tofemale transgender persons $[20,22]$. The reviews were global, collectively including studies from 20 countries. One publication calculated an HIV prevalence of $14.7 \%$ in transgender persons who did not engage in sex work and $27.3 \%$ in those who did [20]. The second review estimated a prevalence of $19.1 \%$ and reported that this population has a 48.8 times higher chance of being infected with HIV than the general population of adults aged between 15 and 49 [22]. We found no reviews on HIV infection in female-to-male transgender persons.

Women who have sex with women (WSW) A low quality systematic review on STIs in WSW globally did not generate any HIV prevalence data in this population [21].

\section{Sexually transmitted infections}

Twelve systematic reviews included data on STIs other than HIV in SGM, including 6 global, 4 regional and 2 national-level reviews [21, 26-36]. Nine of these reviews were on MSM, 2 on WSW and 1 on MSM and transgender persons.

MSM Three reviews reported on syphilis in MSM. Zoni et al. showed high rates of active syphilis prevalence in MSM in Latin America, with rates of $7.5 \%$ or more in half of the included studies [27]. In China, the median syphilis prevalence, based on a review of four studies in MSM, was $14.5 \%$, making MSM the group at highest risk for syphilis in China [28]. Caceres et al. found that syphilis prevalence among MSM in 16 LMICs ranged from $1.5 \%$ in Bangladesh to $29 \%$ in Peru [35].

Hepatitis prevalence was reported in four reviews. A 2003 low quality global systematic review on hepatitis A virus (HAV) did not find an important difference in anti-HAV positivity between MSM and control groups [29]. Two reviews on hepatitis $B$ and $C$ viruses (HBV and $\mathrm{HCV}$ ) in Europe presented disaggregated data for MSM [30, 31]. One showed higher hepatitis C virus antibodies and hepatitis B surface antigen prevalence than in the general population, based on three country estimates [30]. In LMICs, data on HBV prevalence was even sparser. With rates of HBV up to $38 \%$ in Argentina and $31 \%$ in Vietnam, MSM seem to be a high-risk group [35]. A global review comparing incidence of acute HCV in HIV-negative versus HIV-positive MSM found that the incidence in the latter group was four times higher; the incidence rates in HIV-negative MSM were similar

Table 1 Number of reviews selected, by health topic and subpopulation

\begin{tabular}{lllll}
\hline Health topic & $\begin{array}{l}\text { Men who have sex with } \\
\text { men (MSM) }\end{array}$ & $\begin{array}{l}\text { Women who have sex with } \\
\text { women (WSW) }\end{array}$ & $\begin{array}{l}\text { Transgender } \\
\text { persons }\end{array}$ & $\begin{array}{l}\text { Total number } \\
\text { of reviews }\end{array}$ \\
\hline HIV/AIDS & 6 & 1 & 2 & 9 \\
STI & 10 & 2 & 1 & 12 \\
Cancer & 3 & 1 & 0 & 4 \\
Mental health and substance use & 2 & 2 & 0 & 3 \\
Violence & 4 & 2 & 5 & 3 \\
Total & 25 & 8 & 3 & 3 \\
\hline
\end{tabular}


to heterosexual populations [36]. The Pan American Health Organization (PAHO) is currently conducting a review of hepatitis $B$ and $C$ in the countries of the Americas; so far the findings point to a higher burden for MSM (personal communication, Rafael Mazin, focal point for HIV/STIs and hepatitis in key populations, PAHO, 30 May 2014).

In one low quality global review, prevalence of herpes simplex virus 2 (HSV-2) was demonstrated to be higher among male homosexual than among male heterosexual populations [33].

Chlamydia trachomatis and Neisseria gonorrhoeae have not been the subject of global systematic reviews in SGM. One review reported data from nine LMICs with prevalence rates ranging from $0 \%$ (Peru) and $2.1 \%$ (Nepal) up to $14.9 \%$ and $16.1 \%$ (both in Timor-Leste) [35]. A national review on C. trachomatis in Australia designated MSM amongst the populations with the greatest burden, calculating a pooled prevalence for rectal C. trachomatis of $5.6 \%$ [34].

In a high quality global systematic review, Machalek et al. showed a pooled prevalence of any type of anal human papillomavirus (HPV) of $53.6 \%$ in HIV-negative MSM and $89.0 \%$ in HIV-positive MSM [32]. High-grade anal intraepithelial neoplasia was present in $20-30 \%$ of all MSM [32].

WSW Two global systematic reviews on bacterial vaginosis and on STIs in WSW showed a positive association between a history of female sexual partners and risk of bacterial vaginosis $[21,26]$. There was no evidence of a higher burden of STIs in WSW compared with the general population of adult women [21].

Transgender persons Zoni et al. found syphilis prevalence rates ranging from $6.5 \%$ in El Salvador to $43.3 \%$ in Brazil, the latter being the highest rate among highrisk populations (MSM, sex workers and transgender persons) in Latin America and the Caribbean [27]. Preliminary results of an ongoing review on hepatitis $\mathrm{B}$ and $\mathrm{C}$ by PAHO in the region of the Americas show a higher burden for male-to-female transgender persons (personal communication, Rafael Mazin, focal point for HIV/STIs and hepatitis in key populations, PAHO, 30 May 2014).

\section{Cancer}

Four reviews, two high and two low quality, included data on cancer in SGM; all were global in scope [32, 4143]. Three of these reviews were in MSM and 1 in WSW; no reviews were found on cancer burden in transgender persons.
MSM Anal HPV is common in MSM, as are HBV and human herpesvirus $8(\mathrm{HHV}-8)$ infections. These viruses can lead to anal cancer, liver cancer and Kaposi sarcoma, respectively. One review considered MSM at high risk for anal cancer, and found that HIV-positive MSM were at highest risk [43]. Systematic reviews on HPV and on cancer in general in MSM found that HIV-positive MSM had a high incidence of anal cancer, similar to the incidence of cervical cancer in the general female population before the introduction of national cervical screening programmes [32, 41]. Boehmer et al. found that only 1 of the 47 studies included in their review on cancer in MSM was not linked to STIs [41].

WSW Meads \& Moore systematically reviewed studies on breast cancer in lesbian and bisexual women. This review included nine studies with prevalence estimates, of which two showed higher prevalence of breast cancer in lesbian and bisexual women then in heterosexual women, four showed no difference, one showed mixed results and two could not be compared. Though the review was of high quality, all the included studies were small and of poor quality. The reviewers concluded that there was no convincing evidence of a higher disease burden of breast cancer in WSW [42].

\section{Mental health and substance use}

Three high quality reviews -2 global and 1 national provided data on mental health and substance use in SGM [44-46]. Two of these were in MSM and WSW while 1 was in transgender persons.

MSM and WSW In their global review, King et al. calculated an overall 2.47 times increased risk of lifetime suicide attempts in lesbian, gay and bisexual (LGB) people compared to heterosexuals; the risk ratio for lifetime suicidal ideation was 2.04, and it was 2.05 for 12 months prevalence of depression, one of the most common mental illnesses [45]. The reviewers also calculated a pooled risk ratio of 1.88 in gay and bisexual males for 12 months prevalence of anxiety disorders (specific disorders were not specified), but the results for lesbian and bisexual females were less convincing due to heterogeneity in the study methods (I2 $=49.2 \%)$. The review also found that alcohol and substance dependence were at least 1.5 times more common in these populations than in the heterosexual population. Marshal et al. calculated that the odds for substance use among LGB youth globally were $190 \%$ higher than for heterosexual youth [46].

Transgender persons We found data on mental health in transgender persons in a systematic review on the prevalence of HIV and contextual factors potentially 
associated with HIV risk in the United States of America (USA). High rates of suicidal thoughts (weighted mean, $53.8 \%$ ) and lifetime suicide attempts (weighted mean, $31.4 \%)$ were reported in male-to-female transgender persons [44].

\section{Violence}

Violence is one of the more visible health issues in SGM, and the subject of many advocacy efforts. We found four systematic reviews on the prevalence of violence in SGM, including 1 global, 1 regional (North America and Europe) and 2 national-level reviews (USA) [37-40]. All four were on LGB people; there were no reviews on violence in transgender persons.

MSM and WSW A global review found high rates of physical assault (28\%) and sexual assault (27\%) in LGB individuals, and suggested that they experience more victimization than heterosexuals [37]. Peterson et al. concluded that rates of adult sexual assault among gay and bisexual males in North America and Europe far exceed the rates in community samples of men [40]. LGB people in the USA seem to be at increased risk for sexual violence victimization compared with their heterosexual counterparts [38]. Focusing on the subset of intimate partner violence, Finnerman \& Stephenson concluded that emergent evidence in the USA demonstrates that prevalence of intimate partner violence is high in male-male partnerships [39].

\section{Discussion}

Our review found that HIV and STIs are the most researched topics for those studying the disease burden in SGM, and that the subpopulation of MSM is the most intensively studied.

The six included reviews on HIV in MSM (all published in 2007 or later) show a high burden in this population. UNAIDS reports that the median HIV prevalence among MSM exceeds $1 \%$ in all regions of the world and is substantially higher than prevalence among men overall, in every context studied [47]. Furthermore, there is no evidence of a decline in the HIV epidemic in MSM $[18,24,47]$. HIV prevalence among MSM in some lowincome countries could be underreported because the data are too limited; male same-sex sexual behaviour takes multiple forms and HIV has huge potential to increase further in MSM in the next decade [48, 49]. Aburaddad et al. indicated growing - in some cases rapidly growing - HIV epidemics in MSM in several countries, including Egypt, Morocco, Pakistan, Sudan, Tunisia and Yemen [49]. Systematic reviews of the existing research of HIV burden in MSM seem to be up to date and of reasonable quality, though original research and data are still lacking in many countries.
Limited research on HIV in male-to-female transgender persons suggests a high burden. For WSW, a review generated no results but was low in quality [21]. For female-to-male transgender persons and intersex people we found no reviews. The feasibility of conducting a systematic review on disease burden of HIV in these populations should be investigated.

Reviews on STIs reported a high burden of syphilis in MSM in Latin America, China and LMICs where prevalence was measured. A report by the European Surveillance of Sexually Transmitted Infections (ESSTI) network confirms that MSM also bear a disproportionate burden of syphilis across Western Europe and that there is clear evidence that it has increased considerably [50]. Syphilis has been identified as a growing problem among MSM in a number of countries, based on the Global AIDS Response Progress Reports [51].

HAV burden was not found to be higher in MSM compared to heterosexual men in high income countries [29], but a more recent report on viral hepatitis in European MSM did show an elevated prevalence [52]. Increasing attention is being paid to HBV as an STI in MSM. While a 2004, non-sysytematic, review on disease burden of HBV only mentioned homosexual activity as one of the risk factors, alongside heterosexual activity [53], a more recent epidemiological report estimated that HBV incidence among MSM is 20 times higher than in the general population [52]. The same report also looked at HCV, but did not clarify whether its high burden was only in HIV-positive MSM or in MSM in general [52].

A 2014 protocol for a global systematic review on the prevalence of HHV-8 described the investigators' intention to examine the hypothesis that MSM have a high prevalence of the HHV-8 antibody - the infectious disease underlying Kaposi sarcoma - and to determine if the prevalence is increasing [54]. The results have not been published yet.

Our results suggested a high burden of $C$. trachomatis in MSM, based on sparse data from medium quality reviews [34, 35]. Moreover, the most recent annual epidemiological report of the European Centre for Disease Prevention and Control (ECDC), published in 2013, indicated that $99 \%$ of cases of the L2b variant of $C$. trachomatis, which causes the majority of lymphogranuloma venerum infections, are in MSM [55]. Among those cases with known HIV status, 88 \% were HIVpositive.

Data from systematic reviews on the burden of $N$. gonorrhoeae in SGM were too scarce to support any conclusions, but an ESTTI report suggested that MSM bear a disproportionate burden of gonococcal infection in many Western European and some Central and Eastern European countries [50]. The ECDC report also 
indicated that among all $N$. gonorrhoeae cases diagnosed in men in Europe in 2011, 53 \% were in MSM [55]. Moreover, there are indications that MSM are one of the core groups that are key to the emergence, onward transmission and outbreaks of antimicrobial-resistant $N$. gonorrhoeae [56].

The burden of anal HPV is well documented in MSM; the review by Machalek at al. showed high prevalence in MSM in general and very high prevalence in HIV-positive MSM in particular [32]. We found no review on the prevalence of oral HPV, though studies show it is high in MSM, and is a risk factor for oropharyngeal cancer [57].

The systematic review on STI/HIV in WSW did not show a higher burden in this population [21]. Nonetheless, the authors concluded that WSW should not be presumed to be at lower risk based on their sexual orientation, since they can acquire HIV and other STIs through other modes, including injection drug use and sexual contact with high-risk male partners.

Available research on cancer burden in MSM focuses mainly on cancers that are causally linked to STIs; most other cancers have yet to be studied in this subpopulation [41]. Lee et al. showed that there might be reason to believe there are also disparities in other cancers, as sexual minorities in the USA are 1.5 to 2.5 times more likely to be smokers than the heterosexual population [58]. Smoking is a major risk factor for lung cancer and a risk factor for a myriad of other cancers.

Mental health is a poorly reviewed health issue in SGM. The most recent global systematic review on mental health in SGM, although high quality, dated from 2008 and suggested an elevated burden in a variety of mental health issues, but it did not include transgender persons [45]. Eating disorders and obesity were not discussed, although they are mentioned in a review on the health of SGM by the United States Institute of Medicine [9]. Regarding substance use, we found that sexual orientation is an important mediating factor of adolescent substance use [46]. Adding to this, Bourne et al. conducted a global literature review on drug use in MSM and concluded that the prevalence of drug use is high [59]. Some party drugs used by MSM, such as crystal methamphetamine or gamma-butyrolactone, can have detrimental effects on mental and sexual health (an internet survey in Asia reported $4 \%$ and $2.3 \%$ use in MSM, respectively, in the last six months) [59].

Lastly our results show that MSM and WSW are confronted with more violence than their heterosexual counterparts. However, most of the studies were from the USA and other high-income countries; we might expect to see even higher rates of violence in SGM in countries where homosexuality is criminalized. A study from the USA on violence in transgender persons assessed self-report surveys and needs assessments, hotline calls, social service records and police reports [60]. All these sources indicated that violence against transgender persons starts early in life, that they are at risk for multiple types and repeated incidents of violence, particularly sexual violence, and that this threat lasts throughout their lives.

Except for the reviews that reported on cancer and mental health, there are no systematic reviews on noncommunicable diseases in SGM. Nevertheless, there is a growing body of research on the health of SGM. We analysed data from systematic reviews only; therefore the results of research that have not yet been included in a systematic review are not reflected in this article. More extensive reporting, which includes the results of these studies and of studies on risk, contextual and contributing factors of health disparities and specific health issues in SGM, is beyond the scope of this review. Methodologically, this review is limited by the databases we searched and the use of exclusively English search terms on Google Scholar.

\section{Conclusions}

Our review primarily shows that there is a high burden of disease for certain subpopulations of SGM in HIV, STIs, STI-related cancers and mental health conditions, and that they also face high rates of violence. Secondly, our review revealed many knowledge gaps. Those gaps partly stem from a lack of original research, which needs to be addressed, but there is an equally urgent need to conduct high quality systematic and literature reviews to assess what we already know on the disease burden in SGM.

We recommend that systematic reviews on HIV and HPV in MSM be regularly updated. Reviews on herpes in MSM, STIs in WSW, and on mental health, violence and substance use in MSM and WSW, urgently need to be updated. Special attention should be given to violence based on sexual orientation and gender identity. Global reviews on syphilis, hepatitis, $C$. trachomatis and $N$. gonorrhoeae in MSM, and on all of the most common STIs, mental health and violence in the transgender population need to be conducted. In particular, research on female-to-male transgender persons should be considered, as there are virtually no epidemiologic data on the burden of disease in this population. Literature reviews should include publications on the burden of cancers that are not related to infectious causes and other non-communicable diseases in all SGM. It would facilitate this process if health research, surveys and statistical databases systematically included sexual orientation and gender identity as a demographic characteristic, where possible. There is also a geographical research gap since most of the systematic reviews included studies that were all or mostly from high-income countries. New 
reviews could capture research from LMICs by broadening and deepening search strategies. The quality of the systematic should also improve, as less than half were of high quality,

The majority of the currently available systematic reviews are on MSM and the burden of HIV and STIs. This suggests that a substantial portion of the burden of disease in SGM is related to their sexual behaviours, which means it is preventable. WHO and UNAIDS have been building on that knowledge, issuing guidelines, recommendations and tools, including tools for estimating the numbers of specific populations, and for monitoring and evaluating policies and interventions to remediate the burden of HIV and STI in these populations [2, 61-63]. Additional reviews should be conducted on the non-biological factors that contribute to higher disease burden. In order to provide universal access to health-care, more knowledge is needed on the barriers that SGM face in accessing health services, including the attitudes of health-care providers, who are ethically bound to provide good quality services, regardless of the personal history or characteristics of the patient. Understanding these barriers and the additional health risks they impose is crucial to improving the health status of SGM.

\section{Additional files}

\section{Additional file 1: Search strategy, PubMed, sexual and gender}

minorities. (DOCX $105 \mathrm{~kb}$ )

Additional file 2: Summary table of review of systematic reviews.

(XLSX $17 \mathrm{~kb})$

\begin{abstract}
Abbreviations
AIDS: acquired immunodeficiency syndrome; ECDC: European Centre for Disease Control and Prevention; ESSTI: The European Surveillance of Sexually Transmitted Infections; HAV: hepatitis A virus; HBV: hepatitis B virus; HCV: hepatitis C virus; HHV-8: human herpesvirus 8; HIV: human immunodeficiency virus; HPV: human papillomavirus; HSV-2: herpes simplex virus 2; LMIC: low- and middle-income country; MSM: men who have sex with men; PAHO: Pan American Health Organization; SGM: sexual and gender minorities; STI: sexually transmitted infection; UNAIDS: The Joint United Nations Programme on HIV and AIDS; WHO: World Health Organization; WSW: women who have sex with women.
\end{abstract}

\section{Competing interests}

None.

\section{Authors' contributions}

$K B$ and ES performed the literature review. KB, DC, ES, MT, RK and LS conceived of the study, and participated in its design and coordination. KB wrote the manuscript, with the help of DC, LS, RK, IT, MT and ES. All authors read and approved the final manuscript.

\section{Acknowledgements}

The authors would like to thank Tomas Allan for contributing to the search strategy; Jane Patten of Green Ink (UK) for language, technical and copy-editing.

\section{Disclaimer}

Lale Say, Doris Chou, Igor Toskin, Rajat Khosla, Elisa Scolaro and Marleen Temmerman are staff members of the World Health Organization. The authors alone are responsible for the views expressed in this article and they do not necessarily represent the decisions, policy or views of the World Health Organization.

\section{Author details}

${ }^{1}$ Ghent University, Ghent, Belgium. ${ }^{2}$ Department of Reproductive Health and Research, World Health Organization, Geneva, Switzerland. ${ }^{3}$ I.M. Sechenov First Moscow State Medical University, Moscow, Russian Federation.

\section{Received: 5 June 2015 Accepted: 13 January 2016}

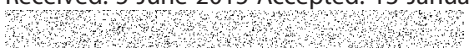

\section{References}

1. Preamble to the Constitution of the World Health Organization as adopted by the International Health Conference, New York, 19-22 June, 1946; signed on 22 July 1946 by the representatives of 61 States, entered into force on 7 April 1948.

2. Prevention and treatment of HIV and other sexually transmitted infections among men who have sex with men and transgender people: recommendations for a public health approach. Geneva: World Health Organization; 2011.

3. UNAIDS terminology guidelines (October 2011). Geneva: Joint United Nations Programme on HIV/AIDS (UNAIDS); 2011

4. Guidelines on estimating the size of populations most at risk to HIV. Geneva: World Health Organization; 2010.

5. Cáceres CF, Konda K, Pecheny M, Chatterjee A, Lyerla R. Estimating the number of men who have sex with men in low and middle income countries. Sex Transm Infect. 2006;82 Suppl 3:iii3-9.

6. Mumtaz G, Hilmi N, McFarland W, Kaplan RL, Akala FA, Semini I, et al. Are HIV epidemics among men who have sex with men emerging in the Middle East and North Africa?: a systematic review and data synthesis. PLoS Med. 2010;8(8):e1000444.

7. Winter S. Lost in transition: transgender people, rights and HIV vulnerability in the Asia-Pacific Region. New York: United Nations Development Programme; 2012

8. Mayer KH, Bradford JB, Makadon HJ, Stall R, Goldhammer H, Landers S. Sexual and gender minority health: what we know and what needs to be done. Am J Public Health. 2008;98(6):989-95.

9. Institute of Medicine, Committee on Lesbian, Gay, Bisexual, and Transgender Health Issues and Research Gaps and Opportunities. The health of lesbian, gay, bisexual, and transgender people: building a foundation for better understanding. Washington (DC): United States National Academy of Sciences; 2011.

10. Bränström R, van der Star A. All inclusive Public Health-what about LGBT populations? Eur J Public Health. 2013;23(3):353-4.

11. Meeting the unique health-care needs of LGBTQ people. Editorial. Lancet. 2016; 387(10014): 95.

12. Boehmer U. Twenty years of public health research: inclusion of lesbian, gay, bisexual, and transgender populations. Am J Public Health. 2002;92(7):1125-30.

13. Bogart LM, Revenson $T$, Whitfield KE, et al. Introduction to the special section on lesbian, gay, bisexual, and transgender (LGBT) health disparities: Where we are and where we're going. Ann Behav Med. 2014;47(1):1-4.

14. Smith V, Devane D, Begley CM, Clarke M. Methodology in conducting a systematic review of systematic reviews of healthcare interventions. BMC Med Res Methodol. 2011;11:15.

15. Becker LA, Oxman AD. Chapter 22: Overviews of reviews. In: Higgins JPT Green S (editors), Cochrane Handbook for Systematic Reviews of Interventions Version 5.1.0 (updated March 2011). The Cochrane Collaboration, 2011. Available from www.cochrane-handbook.org

16. Moher D, Liberati A, Tetzlaff J, Altman DG, The PRISMA Group. Preferred reporting items for systematic reviews and meta-analyses: The PRISMA Statement. PLoS Med. 2009;6(6):e1000097.

17. Shea BJ, Hamel C, Wells GA, Bouter LM, Kristjansson E, et al. AMSTAR is a reliable and valid measurement tool to assess the methodological quality of systematic reviews. J Clin Epidemiol. 2009;62(10):1013-20.

18. Dokubo EK, Kim A, Le L-V, Nadol PJ, Prybylski D, Wolfe MI. HIV incidence in Asia: a review of available data and assessment of the epidemic. AIDS Rev. 2013;15(2):67-76.

19. Miller WM, Buckingham L, Sánchez-Domínguez MS, Morales-Miranda S, PazBailey G. Systematic review of HIV prevalence studies among key populations in Latin America and the Caribbean. Salud Publica Mex. 2013;55 Suppl 1:S65-78.

20. Operario D, Soma T, Underhill K. Sex work and HIV status among transgender women: systematic review and meta-analysis. J Acquir Immune Defic Syndr. 2008;48(1):97-103. 
21. Gorgos LM, Marrazzo JM. Sexually transmitted infections among women who have sex with women. Clin Infect Dis. 2011;53 Suppl 3:S84-91.

22. Baral SD, Poteat T, Strömdahl S, Wirtz AL, Guadamuz TE, Beyrer C. Worldwide burden of HIV in transgender women: a systematic review and meta-analysis. Lancet Infect Dis. 2013;13(3):214-22.

23. Baral S, Sifakis F, Cleghorn F, Beyrer C. Elevated risk for HIV infection among men who have sex with men in low- and middle-income countries 20002006: a systematic review. PLoS Med. 2007;4(12):e339.

24. Beyrer C, Baral SD, van Griensven F, Goodreau SM, Chariyalertsak S, Wirtz AL, et al. Global epidemiology of HIV infection in men who have sex with men. Lancet. 2012;380(9839):367-77.

25. Sullivan PS, Jones JS, Baral SD. The global north: HIV epidemiology in highincome countries. Curr Opin HIV AIDS. 2014;9(2):199-205.

26. Fethers K, Fairley CK, Hocking JS, Gurrin LC, Bradshaw CS. Sexual risk factors and bacterial vaginosis: a systematic review and meta-analysis. Clin Infect Dis. 2008;47(11):1426-35

27. Zoni AC, González MA, Sjögren HW. Syphilis in the most at-risk populations in Latin America and the Caribbean: a systematic review. Int J Infect Dis. 2013;17(2):e84-92.

28. Lin CC, Gao X, Chen X-S, Chen Q, Cohen MS. China's syphilis epidemic: a systematic review of seroprevalence studies. Sex Transm Dis. 2006;33(12):726-36.

29. Franco E, Giambi C, lalacci R, Coppola RC, Zanetti AR. Risk groups for hepatitis A virus infection. Vaccine. 2003;21(19-20):2224-33.

30. Hahné SJM, Veldhuijzen IK, Wiessing L, Lim T-A, Salminen M, Van De Laar M. Infection with hepatitis B and C virus in Europe: a systematic review of prevalence and cost-effectiveness of screening. BMC Infect Dis. 2013;13:181.

31. Hepatitis B and C in the EU neighbourhood: prevalence, burden of disease and screening policies. Stockholm: European Centre for Disease Prevention and Control; 2010

32. Machalek D, Poynten M, Jin F, Fairley CK, Farnsworth A, Garland SM, et al. Anal human papillomavirus infection and associated neoplastic lesions in men who have sex with men: a systematic review and meta-analysis. Lancet Oncol. 2012;13(5):487-500.

33. Smith JS, Robinson NJ. Age-specific prevalence of infection with herpes simplex virus types 2 and 1: a global review. J Infect Dis. 2002;186(Suppl):S3-28.

34. Lewis D, Newton DC, Guy RJ, Ali H, Chen MY, Fairley CK, et al. The prevalence of Chlamydia trachomatis infection in Australia: a systematic review and meta-analysis. BMC Infect Dis. 2012;12:113.

35. Cáceres CF, Konda K, Segura ER, Lyerla R. Epidemiology of male same-sex behaviour and associated sexual health indicators in low- and middleincome countries: 2003-2007 estimates. Sex Transm Infect. 2008:84(Suppl I):i49-56.

36. Yaphe S, Bozinoff N, Kyle R, Shivkumar S, Pai NP, Klein M. Incidence of acute hepatitis $C$ virus infection among men who have sex with men with and without HIV infection: a systematic review. Sex Transm Infect. 2012:88(7):558-64.

37. Katz-Wise SL, Hyde JS. Victimization experiences of lesbian, gay, and bisexual individuals: a meta-analysis. J Sex Res. 2012:49(2-3):142-67.

38. Rothman EF, Baughman A. The prevalence of sexual assault against people who identify as gay, lesbian or bisexual in the United States: a systematic review. Trauma Violence Abuse. 2012;12(2):55-66.

39. Finneran C, Stephenson R. Intimate partner violence among men who have sex with men: a systematic review. Trauma Violence Abuse. 2013;14(2):168-85.

40. Peterson ZD, Voller EK, Polusny M, Murdoch M. Prevalence and consequences of adult sexual assault of men: review of empirical findings and state of the literature. Clin Psychol Rev. 2011;31(1):1-24.

41. Boehmer U, Cooley TP, Clark M. Cancer and men who have sex with men: a systematic review. Lancet Oncol. 2012;13(12):e545-53.

42. Meads C, Moore D. Breast cancer in lesbians and bisexual women: systematic review of incidence, prevalence and risk studies. BMC Public Health. 2013:13:1127.

43. Van der Zee RP, Richel O, de Vries HJC, Prins JM. The increasing incidence of anal cancer: can it be explained by trends in risk groups? Neth J Med. 2013;71(8):401-11.

44. Herbst JH, Jacobs ED, Finlayson TJ, McKleroy VS, Neumann MS, Crepaz N. Estimating HIV prevalence and risk behaviors of transgender persons in the United States: a systematic review. AIDS Behav. 2008;12(1):1-17.

45. King M, Semlyen J, Tai SS, Killaspy H, Osborn D, Popelyuk D, et al. A systematic review of mental disorder, suicide, and deliberate self harm in lesbian, gay and bisexual people. BMC Psychiatry. 2008:8:70.
46. Marshal MP, Friedman MS, Stall R, King KM, Miles J, Gold M, et al. Sexual orientation and adolescent substance use: a meta-analysis and methodological review. Addiction. 2008;103(4):546-56.

47. Global report: UNAIDS report on the global AIDS epidemic 2013. Geneva: Joint United Nations Programme on HIV/AIDS; 2013

48. Van Griensven F, de Lind van Wijngaarden JW. A review of the epidemiology of HIV infection and prevention responses among MSM in Asia. AIDS. 2010;24 Suppl 3:S30-40.

49. Abu-raddad L, Akala FA, Semini I, Riedner G, Wilson D, Tawil O. HIV/AIDS Epidemic in the Middle East and North Africa: time for strategic action. Washington (DC): World Bank; 2010.

50. Savage EJ, Hughes G, Ison C, Lowndes CM; The European Surveillance of Sexually Transmitted Infections (ESSTI) network. Syphilis and gonorrhoea in men who have sex with men: a European overview. Euro Surveill. 2009;14(47).

51. Report on global sexually transmitted infection surveillance 2013. Geneva: World Health Organization; 2014

52. Urbanus AT, van Houdt R, van de Laar TJ, Coutinho RA. Viral hepatitis among men who have sex with men, epidemiology and public health consequences. Euro Surveill. 2009;14(47)

53. Custer B, Sullivan SD, Hazlet TK, Iloeje U, Veenstra DL, Kowdley KV. Global epidemiology of hepatitis B virus. J Clin Gastroenterol. 2004;38 Suppl 3:S158-68.

54. Rohner E, Wyss N, Trelle S, Mbulaiteye SM, Egger M, Novak U, et al. HHV-8 seroprevalence: a global view. Syst Rev. 2014;3(1):11.

55. Annual Epidemiological Report 2013. Reporting on 2011 surveillance data and 2012 epidemic intelligence data. Stockholm: European Centre for Disease Prevention and Control; 2013

56. Lewis DA. The role of core groups in the emergence and dissemination of antimicrobial-resistant N. gonorrhoeae. Sex Transm Infect. 2013;89 Suppl 4:iv47-51.

57. Mooij SH, Boot HJ, Speksnijder AGCL, Stolte IG, Meijer CJLM, Snijders PJF, et al. Oral human papillomavirus infection in HIV-negative and HIV-infected men who have sex with men. AIDS. 2013;27(13):2117-28

58. Lee JG, Griffin GK, Melvin CL. Tobacco use among sexual minorities in the USA, 1987 to May 2007: a systematic review. Tob Control. 2009;18:275-82.

59. Bourne A. Drug use among men who have sex with men: implications for harm reduction. In: Stoicescu C, editor. Global State of Harm Reduction 2012. London: Harm Reduction International; 2012. p. 147-55.

60. Stotzer RL. Violence against transgender people: a review of United States data. Aggress Violent Behav. 2009;14(3):170-9.

61. Consolidated guidelines on HIV prevention, diagnosis, treatment and care for key populations. Geneva: World Health Organization; 2014.

62. Operational guidelines for monitoring and evaluation of HIV programmes for sex workers, men who have sex with men and transgender people: national and sub-national level. Geneva: Joint United Nations Programme on HIV/AIDS (UNAIDS); 2013.

63. Tool for setting and monitoring targets for HIV prevention diagnosis, treatment and care for key populations. Geneva: World Health Organization; 2015. (in press)

\section{Submit your next manuscript to BioMed Central and we will help you at every step:}

- We accept pre-submission inquiries

- Our selector tool helps you to find the most relevant journal

- We provide round the clock customer support

- Convenient online submission

- Thorough peer review

- Inclusion in PubMed and all major indexing services

- Maximum visibility for your research

Submit your manuscript at www.biomedcentral.com/submit 